\title{
Flow analysis of particulate suspension on an asymmetric peristaltic motion in a curved configuration with heat and mass transfer
}

\author{
Ahmed Zeeshan ${ }^{1, *}$, Nouman Ijaz ${ }^{1}$, and Muhammad Mubashir Bhatti ${ }^{2}$ \\ ${ }^{1}$ Department of Mathematics and Statistics, FBAS, International Islamic University, Islamabad, Pakistan \\ ${ }^{2}$ Shanghai Institute of Applied Mathematics and Mechanics, Shanghai University, Shanghai 200072, PR China
}

Received: 31 October 2017 / Accepted: 9 April 2018

\begin{abstract}
This article addresses the influence of particulate-fluid suspension on asymmetric peristaltic motion through a curved configuration with mass and heat transfer. A motivation for the current study is that such kind of theory is helpful to examine the two-phase peristaltic motion between small muscles during the propagation of different biological fluids. Moreover, it is also essential in multiple applications of pumping fluid-solid mixtures by peristalsis, i.e., Chyme in small intestine and suspension of blood in arteriole. Long wavelength, as well as small Reynolds number, have been utilized to render the governing equations for particle and fluid phase. Exact solutions are presented for velocity $\left(u_{f, p}\right)$, temperature $\left(\theta_{f, p}\right)$ and concentration distributions $\left(\varphi_{f, p}\right)$. All the parameters such as Prandtl number $\left(P_{r}\right)$, particle volume fraction $(C)$, suspension parameter $\left(M_{1}\right)$, curvature parameter $(k)$, volumetric flow rate $(Q)$, Schmidt number $\left(S_{c}\right)$, phase difference $(\varphi)$, Eckert number $\left(E_{c}\right)$, and Soret number $\left(S_{r}\right)$ discussed graphically for peristaltic pumping $(\Delta p)$, pressure gradient $(d p / d x)$, velocity $\left(u_{f, p}\right)$, temperature $\left(\theta_{f, p}\right)$ and concentration distributions $\left(\varphi_{f, p}\right)$. The streamlines are also plotted with the aid of contour.
\end{abstract}

Keywords: Peristaltic flow / particle-fluid / curved channel / mass and heat transfer / exact solutions

\section{Introduction}

The physiology of peristaltic flow ("sinusoidal motion") grabs the concentration of many researchers because of its multiple applications in biomedical engineering and industry. This process is substantial in the various process involved in a human body, i.e., propagation cilia wave, a motion of spermatozoa, vasomotion of small blood vessels, Chyme propagation, and urine propagation from a kidney to bladder, etc. This phenomenon is wholly associated with finger and roller pumps, the heart-lung machine, and circulation of toxic and sanitary liquid. Due to these incredible and vital applications of peristaltic flow, many authors explored this phenomenon using a viscous fluid model in different geometrical situations. Mishra and Rao [1] discussed the asymmetric peristaltic motion of Newtonian fluid model propagating through a finite channel. They used the lubrication approach to model the governing equations and obtained the exact solutions. Nonlinear peristaltic motion of viscous fluid propagating through a porous medium was examined by Mekheimer [2]. The

\footnotetext{
* e-mail: ahmad.zeeshan@iiu.edu.pk
}

three-dimensional flow with a viscous fluid model was studied by Reddy et al. [3]. Later, Elnaby and Haroun [4] presented a new model to analyze the wall behavior of inclined planar channel. The finite planar channel filled with a viscous fluid model and porous walls. Hayat et al. [5] analyzed the peristaltic flow of viscous fluid in a curved compliant channel. Ramanamurthy et al. [6] discussed the unsteady peristaltic motion of viscous fluid in the curved channel. They also used long wavelength and small Reynolds approximation and presented the exact solutions. Recently, Nadeem et al. [7] extended the work of Reddy et al. [3] and explored the behavior of viscous fluid propagating in a three-dimensional duct with compliant walls.

The presence of particles in fluids is a ubiquitous phenomenon because it involves deeply in our daily life. For instance, the water we drink, the air we inhale, and fizzy drinks involve many particles in different forms. Moreover, blood an essential element promptly arises in our mind that occurs with white blood cells, plasma, and red blood cells. There are multiple numbers of examples that include the collision between fluid and particles that can be encountered in our daily life. According to the technological standpoint, various activates in the industrial process 
include particle-fluids systems, i.e., fluidization, powder technology, lunar ash flow, sedimentation, aerosol filtration, and combustion, etc. Such kind of theory is also beneficial to analyze the hydrodynamics of biological systems because it gives better information in blood rheology, electrophoresis, dethroning of particles in the respiratory tract, separation of chromatographic, diffusion of proteins and macromolecules separation process. Multiphase flows are of multiple types, i.e., Gas-liquid flows (i.e., gas-droplet flows, separated flows and bubbly flows), Gas-solid flows (i.e., Gas-particle flows, Pneumatic motion, and Fluidized beds) and solid-liquid flows (i.e., Slurry flows, Sediment motion and Hydro transport). Various researchers have examined the peristaltic movement having solid particles in different geometrical situations. Misra and Pandey [8] discussed the peristaltic movement through a cylindrical tube having small spherical particles of similar size. They obtained the solution using perturbation technique and observed the converse flow appearance when a pressure gradient is higher than the critical value and it occurs because of the occupancy of particles. Saxena and Srivastava [9] addressed the particulate suspension peristaltic flow through a non-uniform axisymmetric tube. Mekheimer et al. [10] investigated the sinusoidal motion particles through a planar channel. Recently, Mekheimer et al. [11] again considered the eccentric cylinders with thread annular and sinusoidal waves traveling containing small particles. Kamel et al. [12] investigated the peristaltic motion through a planar channel having small particles in it. Few more studies are available in references [13-16].

Mass and heat transfer effects on peristaltic flow also have multiple applications in industry and biomedical science. Heat transfer includes multiple complex processes in tissues, i.e., heat conduction, heat convection because of the hemodynamics ("blood flow") and radiation process. The mass transfer also has a valuable role in all these processes. For instance, oxygenation and hemodialysis process has been examined by taking the peristaltic flow with heat transfer. A complex relationship arises between driving potentials and fluxes in the presence of combined mass and heat transfer effects. The energy flux persuaded by composition gradients and temperature gradient whereas mass flux can be originated by a temperature gradient (i.e., "soret effect"). The mass transfer has a remarkable role in the diffusion process, i.e., diffusion of nutrients from the blood to neighboring tissues. Mass and heat transfer also arises in between fluid and solid particles and flowing Newtonian/non-Newtonian media, i.e., multiphase fluidized bed systems, bubble columns and boundary layer flows. Furthermore, it can also encounter in the manufacturing of polymer alloys (via "liquid phase route"), devolatilization of films and food processing, etc. Mass and heat transfer process on Newtonian fluid models provides essential knowledge for single particles of highly romanticize geometry, i.e., cylinder, curved channels, flat plate, etc. but it becomes more complicated in multiparticle flows. Mass and heat transfer process arises in multiple geometrical aspects with different boundary conditions examined extensively by various authors. Tripathi [17] analyzed the heat transfer effects on the peristaltic motion of food bolus through an oesophagus and presented a new mathematical model. Further, they examined numerically the behavior of two inherent mechanisms i.e., reflux and trapping in the presence of heat transfer. Srinivas et al. [18] described the behavior of heat and mass transfer on the asymmetric peristaltic propulsion of Newtonian fluid in the presence of slip impact. The three-dimensional nonuniform peristaltic motion of a viscous fluid with heat and mass transfer through a rectangular duct was examined by Ellahi et al. [19]. Bhatti et al. [20] studied the heat transfer on peristaltic blood flow containing small particles propagating in a non-uniform tube. They further assumed the variable viscosity of the blood and presented the exact results against particle and fluid phase. Recently, Bhatti et al. [21] studied the behavior of heat and mass transfer on the peristaltically induced motion of particlefluid simultaneously through a finite channel with slip effects. Again, Bhatti et al. [22] discussed the EMHD and non-linear thermal radiation on peristaltic motion of viscous dusty fluid through a porous medium.

To the best of our knowledge, no effort has been devoted to estimate the mass and heat transfer influence on the asymmetric peristaltic motion of viscous fluid having small particles through a curved channel. In the previous studies, it is found that different authors have discussed particlefluid suspension, but asymmetric/symmetric peristaltic motion in the presence of small particles in curved configuration was not discussed by anyone. To fill this gap, the present study has been presented. Consequently, a remarkable effort has hitherto been dedicated to develop a better understanding of the two-phase asymmetric peristaltic motion of viscous fluid in the presence of mass and heat transfer. The inspiration of current study is that the theory of multiphase flow is beneficial to analyze the sinusoidal muscular expansion and contraction in propagating of various biological fluids. The present flow is modeled with a long wavelength and ignoring the inertial forces. Closed form solutions are obtained for particle and fluid phase.

\section{Problem modelling}

Let us consider an asymmetric peristaltic motion of a viscous fluid with incompressible, constant density and irrotational properties having small particles propagating with constant celerity. The geometry of walls (see Figure 1) is described as

Upper wall : $h_{1}(\bar{x}, \bar{t})=a_{1} \cos 2 \pi \lambda^{-1}[\bar{x}-c \bar{t}]+d_{1}$,

Lower wall : $h_{2}(\bar{x}, \bar{t})=b_{1} \cos 2 \pi \lambda^{-1}[\bar{x}+\Theta-c \bar{t}]-d_{2}$.

In above equation, the phase difference $\Theta$ having range $0 \leq \Theta \leq \pi$ whereas $\Theta=\pi$ is associated with a wave in a phase and $\Theta=0$ related to symmetric channel having waves out of phase. The other constants satisfy the following condition.

$$
a_{1}^{2}+b_{1}^{2}+2 a_{1} b_{1} \cos \leq\left(d_{1}+d_{2}\right)^{2} .
$$




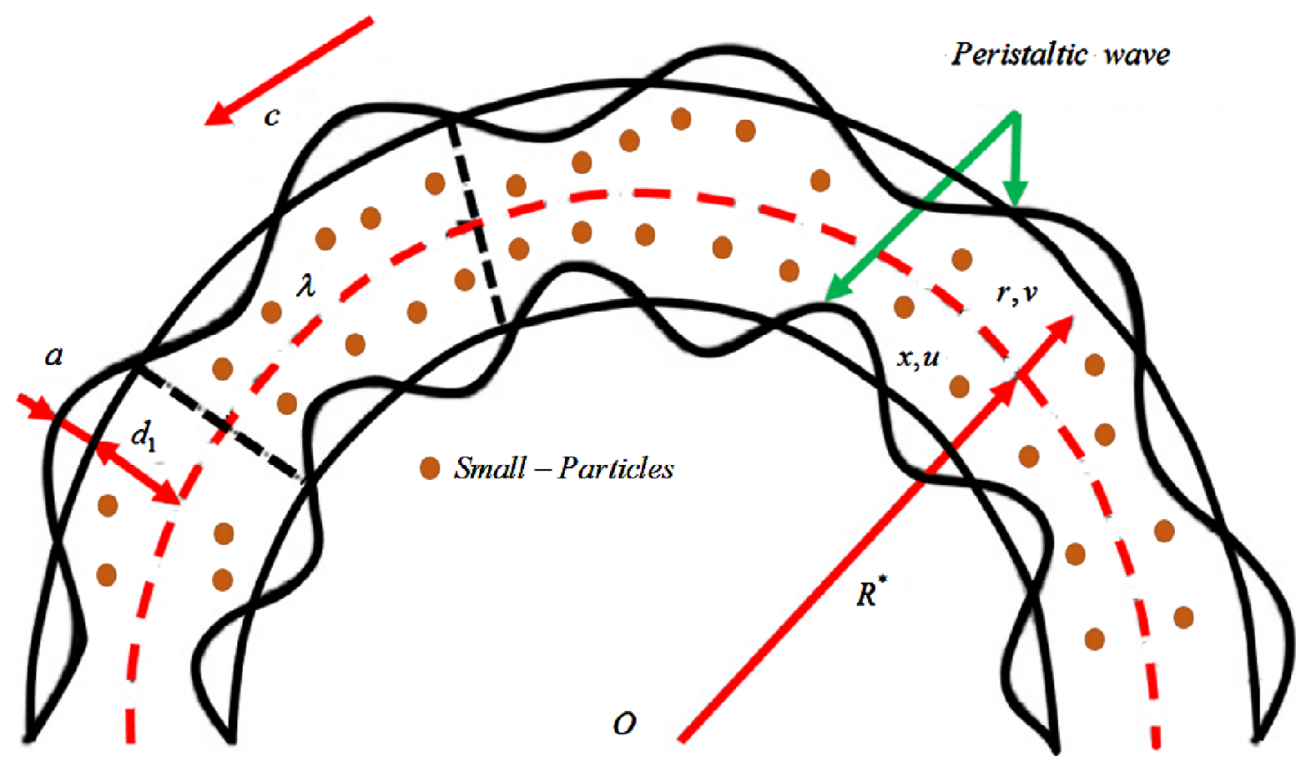

Fig. 1. Flow structure.

\subsection{Fluid phase}

$$
\begin{gathered}
\frac{\partial\left[\left(r+R^{*}\right) v_{f}\right]}{\partial r}+R^{*} \frac{\partial u_{f}}{\partial x}=0 \\
(1-C) \rho_{f}\left(\frac{\partial v_{f}}{\partial t}+v_{f} \frac{\partial v_{f}}{\partial r}+\frac{u_{f} R^{*}}{r+R^{*}} \frac{\partial v_{f}}{\partial x}+\frac{u_{f}^{2}}{r+R^{*}}\right) \\
=-(1-C) \frac{\partial P}{\partial r}+\frac{2 \mu_{s}(1-C)}{\left(r+R^{*}\right)^{2}} \frac{\partial}{\partial r}\left[\frac{\partial v_{f}}{\partial r}\left(R^{*}+r\right)\right] \\
+\frac{\mu_{s}\left(R^{*}-R^{*} C\right)}{\left(R^{*}+r\right)} \frac{\partial}{\partial x}\left(\frac{\partial u_{f}}{\partial r}+\frac{R^{*}}{R^{*}+r} \frac{\partial v_{f}}{\partial x}-\frac{u_{f}}{R^{*}+r}\right) \\
-(1-C)\left(\frac{2 \mu_{s}}{r+R^{*}}\right)\left(\frac{R^{*}}{R^{*}+r} \frac{\partial u_{f}}{\partial x}+\frac{v_{f}}{R^{*}+r}\right) \\
+C S^{\prime}\left(v_{p}-v_{f}\right), \\
(1-C) \rho_{f}\left(\frac{\partial u_{f}}{\partial t}+v_{f} \frac{\partial u_{f}}{\partial r}+\frac{R^{*}}{R^{*}+r} u_{f} \frac{\partial u_{f}}{\partial x}+\frac{u_{f} v_{f}}{r+R^{*}}\right) \\
=-\frac{R^{*}(1-C)}{r+R^{*}} \frac{\partial P}{\partial x}+\frac{\mu_{s}(1-C)}{\left(r+R^{*}\right)^{2}} \frac{\partial}{\partial r} \\
{\left[\frac{\partial u_{f}}{\partial r}\left(R^{*}+r\right)^{2}\right]+(1-C) \frac{\mu_{s}}{\left(r+R^{*}\right)^{2}} \frac{\partial}{\partial r}} \\
{\left[\left(\frac{R^{*}}{R^{*}+r} \frac{\partial v_{f}}{\partial x}-\frac{u_{f}}{r+R^{*}}\right)\left(R^{*}+r\right)^{2}\right]} \\
+(1-C)\left(\frac{2 \mu_{s} R^{*}}{R^{*}+r}\right) \frac{\partial}{\partial x}\left[\left(\frac{\partial u_{f}}{\partial x} \frac{R^{*}}{\left(R^{*}+r\right)}+\frac{v_{f}}{\left(R^{*}+r\right)}\right)\right] \\
+C S^{\prime}\left(u_{p}-u_{f}\right),
\end{gathered}
$$$$
(1-C) \rho_{f} C_{p}\left(\frac{\partial T_{f}}{\partial t}+v_{f} \frac{\partial T_{f}}{\partial r}+\frac{u_{f} R^{*}}{r+R^{*}} \frac{\partial T_{f}}{\partial x}\right)=K(1-C)
$$$$
\left(\frac{\partial^{2} T_{f}}{\partial r^{2}}+\frac{1}{R^{*}+r} \frac{\partial T_{f}}{\partial r}+\left(\frac{R^{*}}{R^{*}+r}\right)^{2} \frac{\partial^{2} T_{f}}{\partial x^{2}}\right)
$$$$
+\frac{\rho_{f} C_{p} C\left(T_{p}-T_{f}\right)}{\varpi_{T}}+\frac{C S^{\prime}\left(u_{f}-u_{p}\right)^{2}}{\varpi_{r}},
$$

$$
\begin{gathered}
\left(\frac{\partial^{2} F_{f}}{\partial r^{2}}+\frac{1}{R^{*}+r} \frac{\partial F_{f}}{\partial r}+\frac{\partial^{2} F_{f}}{\partial x^{2}}\left(\frac{R^{*}}{R^{*}+r}\right)^{2}\right)+\frac{D_{m}}{T_{m}}\left(k_{T}-k_{T} C\right) \\
\left(\frac{\partial^{2} T_{f}}{\partial r^{2}}+\frac{1}{r+R^{*}} \frac{\partial T_{f}}{\partial r}+\left(\frac{R^{*}}{r+R^{*}}\right)^{2} \frac{\partial^{2} T_{f}}{\partial x^{2}}\right) \\
+\frac{\rho_{f} C_{p} C S^{\prime}\left(F_{p}-F_{f}\right)}{\varpi_{c}},
\end{gathered}
$$

\subsection{Particulate phase}

$$
\begin{gathered}
\frac{\partial\left[\left(R^{*} v_{p}+r v_{p}\right)\right]}{\partial r}+\frac{\partial u_{p}}{\partial x} R^{*}=0 \\
\rho_{p}\left(C \frac{\partial v_{p}}{\partial t}+C \frac{\partial v_{p}}{\partial r} v_{p}+C \frac{u_{p} R^{*}}{R^{*}+r} \frac{\partial v_{p}}{\partial x}+C \frac{u_{p}^{2}}{r+R^{*}}\right) \\
=-C \frac{\partial P}{\partial r}-S^{\prime} C\left(v_{p}-v_{f}\right), \\
\rho_{p}\left(C \frac{\partial u_{p}}{\partial t}+v_{p} C \frac{\partial u_{p}}{\partial r}+\frac{R^{*} C}{R^{*}+r} u_{p} \frac{\partial u_{p}}{\partial x}+\frac{u_{p} C v_{p}}{R^{*}+r}\right) \\
=-\frac{R^{*} C}{R^{*}+r} \frac{\partial P}{\partial x}+S^{\prime} C\left(u_{f}-u_{p}\right), \\
\rho_{f} C_{p} C\left(\frac{\partial T_{p}}{\partial t}+v_{p} \frac{\partial T_{p}}{\partial r}+\frac{u_{p} R^{*}}{r+R^{*}} \frac{\partial T_{p}}{\partial x}+\frac{u_{p} v_{p}}{r+R^{*}}\right) \\
=\frac{\rho_{f} C_{p} C\left(T_{p}-T_{f}\right)}{\varpi_{T}},
\end{gathered}
$$




$$
\begin{aligned}
\rho_{p} C\left(\frac{\partial F_{p}}{\partial t}+v_{p} \frac{\partial F_{p}}{\partial r}+\frac{R^{*}}{r+R^{*}} u_{p} \frac{\partial F_{p}}{\partial x}+\frac{u_{p} v_{p}}{r+R^{*}}\right) \\
=\frac{\rho_{p} C_{p} C\left(F_{f}-F_{p}\right)}{\varpi_{c}} .
\end{aligned}
$$

where

$$
\left.\begin{array}{l}
S^{\prime}=\frac{9 \mu_{0}}{2 a^{2}} \lambda(C) \\
\lambda(C)=\left[3 \sqrt{-3 C^{2}+8 C}+4+3 C\right](2-3 C)^{-2} \\
\mu_{s}=\frac{\mu_{0}}{1-\chi C^{\prime}} \\
\left.\chi=0.07 e^{C\left[\frac{1107}{C T e^{1.69 C}}+2.49\right.}\right]
\end{array}\right\}
$$

To move from fixed frame to wave frame, let us define the following variables as

$$
\begin{aligned}
x^{*} & =x-c t, r^{*}=r, z^{*}=z, v^{*}=v, u^{*} \\
& =u-c p\left(x^{*}, z^{*}\right)=P(x, z, t) .
\end{aligned}
$$

Henceforward, the dimensionless quantities are

See equation 16 below page

Using equation (15) and equation (16) in equations (3)(13), and assuming creeping flow with a long wavelength, the resulting equations for fluid phase can be written as

$$
\begin{aligned}
& \frac{\mathrm{d} p}{\mathrm{~d} x}=\left(\frac{k+r}{k}\right)\left(\frac{1}{k+r} \frac{\partial}{\partial r}\left(\frac{\partial u_{f}}{\partial r}(k+r)^{2}\right)\right. \\
& \left.\quad+\frac{\partial}{\partial r}\left(-\frac{u_{f}}{r+k}(k+r)^{2}\right)\right)+M_{1} C\left(u_{p}-u_{f}\right), \\
& \frac{\partial^{2} \theta_{f}}{\partial r^{2}}+\frac{1}{(k+r)} \frac{\partial \theta_{f}}{\partial r}=-P_{r} M_{1}\left(\theta_{p}-\theta_{f}\right) \\
& \quad-P_{r} E_{c} M_{1} C\left(u_{p}-u_{f}\right)^{2}, \\
& \frac{\partial^{2} \phi_{f}}{\partial r^{2}}+\frac{1}{(k+r)} \frac{\partial \phi_{f}}{\partial r}+N_{A}\left(\frac{\partial^{2} \theta_{f}}{\partial r^{2}}+\frac{1}{(r+k)} \frac{\partial \theta_{f}}{\partial r}\right) \\
& =-C M_{1}\left(\phi_{p}-\phi_{f}\right)
\end{aligned}
$$

and for particulate phase

$$
\begin{gathered}
\frac{\mathrm{d} p}{\mathrm{~d} x}=(1-C) M_{1}\left(u_{f}-u_{p}\right), \\
\theta_{p}=\theta_{f}, \\
\phi_{p}=\phi_{f},
\end{gathered}
$$

Corresponding non-dimensional boundary conditions are

$$
\left.\begin{array}{l}
u_{f}\left(\eta_{1}\right)=-1 \\
u_{f}\left(\eta_{2}\right)=-1
\end{array}\right\}
$$

where

$$
\left.\begin{array}{l}
\eta_{1}(x)=1+a \cos (x) \\
\eta_{2}(x)=-b-d \cos (x+\Theta)
\end{array}\right\}
$$

The exact solutions for fluid and particle phase are obtained as

See equation (25) and (26) below page.

$$
\begin{gathered}
\theta_{f, p}=\theta_{0}+\theta_{1} \log [k+r]+\theta_{2} r+\theta_{3} r^{2}, \\
\phi_{f, p}=\phi_{0}+\phi_{1} \log [k+r]+\phi_{2} r+\phi_{3} r^{2} .
\end{gathered}
$$

The constants with subscripts are defined in the appendix section.

The rate of volume flow is given by

$$
Q=\int_{\eta_{1}}^{\eta_{2}} u_{f}(1-C) d r+\int_{\eta_{1}}^{\eta_{2}} u_{p} C d r
$$

The pressure gradient can be evaluated with the help of above expression and we have

$$
\begin{gathered}
x=\frac{x^{*}}{\lambda}, r=\frac{r^{*}}{d}, u=\frac{u^{*}}{c}, v=\frac{v^{*}}{c}, p^{*}=\frac{d^{2} p}{\mu c \lambda}, \delta=\frac{d}{\lambda}, k=\frac{R^{*}}{d}, h_{1}^{*}=\frac{h_{1}}{d_{1}}, P_{r}=\frac{\mu C}{K}, N_{A}=S_{r} S_{c}, \\
h_{2}^{*}=\frac{h_{2}}{d_{1}}, d^{*}=\frac{d_{2}}{d_{1}}, a^{*}=\frac{a_{1}}{d_{1}}, b^{*}=\frac{b_{1}}{d_{1}}, R e=\frac{\rho_{f} d c \delta}{\mu}, \theta_{f, p}=\frac{T_{f, p} T_{0}}{T_{1} T_{0}}, \phi_{f, p}=\frac{F_{f, p} F_{0}}{F_{1} F_{0}}, \\
E_{c}=\frac{C^{2}}{C\left(T_{1} T_{0}\right)}, M_{1}=\frac{S^{\prime} a^{2}}{(1 C) \mu_{s}}, S_{c}=\frac{\mu_{s}}{\rho D_{m}}, S_{r}=\frac{\rho D_{m} k_{T}\left(T_{1} T_{0}\right)}{\mu_{s} T_{m}\left(F_{1} F_{0}\right)} . \\
u_{f}=\frac{u_{0}+u_{1} r+u_{2} r^{2}+u_{3} r^{3}+u_{4} \log [r+k]+u_{5} r \log [r+k]+u_{6} r^{2} \log [r+k]}{r+k}, \\
u_{p}=\frac{u_{0}+u_{1} r+u_{2} r^{2}+u_{3} r^{3}+u_{4} \log [r+k]+u_{5} r \log [r+k]+u_{6} r^{2} \log [r+k]}{r+k} \frac{1}{(1 C) M_{1}} \frac{\mathrm{d} p}{\mathrm{~d} x},
\end{gathered}
$$



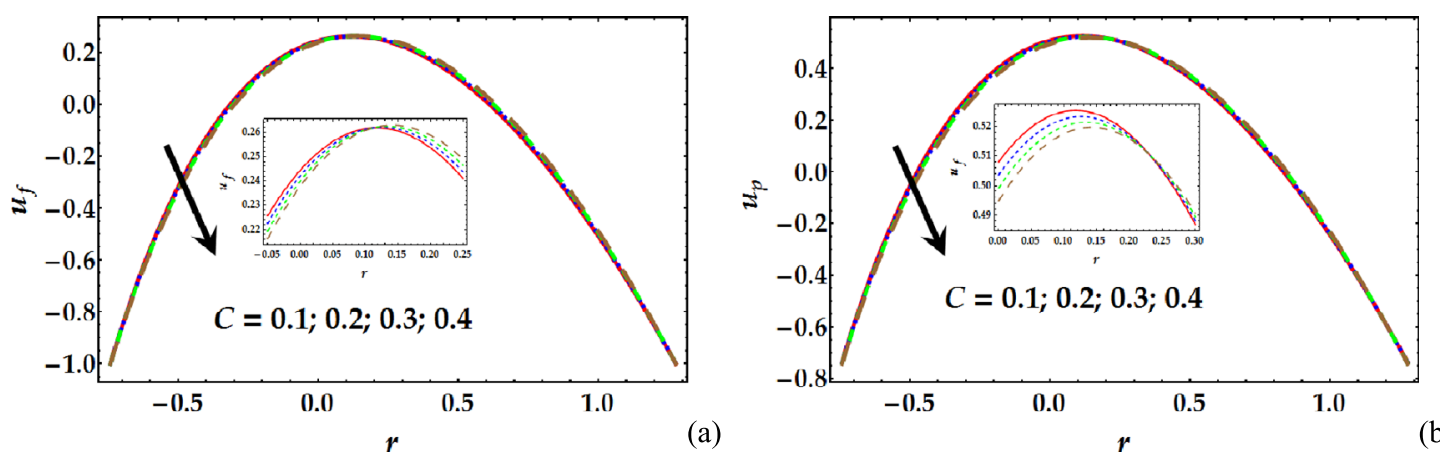

Fig 2. Velocity curves for multiple values of $C$.

See equation (30) below page.

The pressure rise $\Delta p$ in dimensionless form is defined as

$$
\Delta p=\int_{0}^{1} \frac{\mathrm{d} p}{\mathrm{~d} x} d x .
$$

\section{Illustrations and physical discussion}

The aim of this section is to see the variation of different parameters on peristaltic pumping $(\Delta p)$, streamlines, velocity $\left(u_{f, p}\right)$, pressure gradient $(d p / d x)$, temperature $\left(\theta_{f, p}\right)$ and concentration distributions $\left(\varphi_{f, p}\right)$. Numerical computation is used by means built-in "shooting algorithm" in "Mathematica (10.3v)" software. Particularly, we explored the variation of all the physical quantities i.e., particle volume fraction " $C$ ", suspension parameter " $M_{1}$ ", curvature parameter " $k$ ", volumetric flow rate " $Q$ ", phase difference " $\Theta$ ", Prandtl number " $P_{r}$ ", Eckert number " $E_{c}$ ", Schmidt number " $S_{c}$ " and Soret number " $S$ ", respectively. It is worth to point out here that the current analysis is also applicable for single phase flow by considering " $C=0$ ".

\subsection{Velocity behaviour and pumping characteristics}

Figure $2 \mathrm{a}$ and $\mathrm{b}$ depicts the variation of particle volume fraction " $C$ " on velocity profiles. It reveals from both figures that due to increment in the value of " $C$ ", the variation in velocity is very small, however, the velocity of the fluid acts oppositely near the curvy walls. In the presence of solid particles, the drag force rises and there is retardation in the flow. Moreover, for the other side of the channel $(r>0.1)$, the wall is asymmetric, and the drag force is less effective. The current results are also incorporated with previous studies Bhatti and Zeeshan [23], Kamel et al. [12]. More details are presented in Drew [23]. Figure 3a and b indicates that large values of curvature parameter " $k$ " tend to resist the flow markedly whereas on the opposite of the wall $(r>0.1)$, it fails to produce a significant resistance and as a result, the velocity of the fluid rises. Figure $4 \mathrm{a}$ and $\mathrm{b}$ and Figure 5 present pressure gradient profiles which are plotted with the help of equation (29). It is observed from Figure $4 \mathrm{a}$ and b that volumetric flow rate " $Q$ " reveals completely adverse attitude as compared to particle volume fraction " $C$ " on pressure gradient. It is observed that higher flow rate causes less resistance in the flow and as a result, more pressure is required to control the flow. However, for particle volume fraction " $C$ ", pressure gradient significantly declines due to the higher rate of drag force in the flow. In Figure 5 it is found that when the phase difference " $\Theta$ " increases then pressure gradient also increases. Figure $6 \mathrm{a}$ and $\mathrm{b}$ is drawn to visualize the pumping features. This is an important mechanism in a human body that is very supportive to move various biological fluids (i.e., cilia motion, blood pumping and transport of urine) in a human body. Moreover, in biomedical engineering numerous devices are manufactured on the phenomena of peristaltic pumping. These figures are divided into four regions i.e., peristaltic pumping $\{\Delta p>0, Q>0\}$, retrograde pumping $\{\Delta p>0$, $Q<0\}$, co-pumping $\{\Delta p<0, Q>0\}$ and free pumping $\{\Delta p<0, Q<0\}$. In Figure $6 \mathrm{a}$ it is observed that the magnitude of pumping rate is very high in peristaltic pumping region as well as in retrograde pumping and increases for higher values of particle volume fraction " $C$ ". However, its attitude becomes reverse at $Q=0.6$ and reveals opposite influence in the co-pumping region. It is clear from Figure $6 \mathrm{~b}$ that suspension parameter " $M_{1}$ " significantly enhances the pressure rise in retrograde and peristaltic pumping regions while its behaviour becomes converse at $Q=0.5$ and depicts opposite behaviour in the co-pumping region.

$$
\begin{aligned}
\frac{\mathrm{d} p}{\mathrm{~d} x}= & \frac{36(1+C) M_{1}\left(\eta_{1} \eta_{2}\right)\left[\left(\eta_{2}+Q \eta_{1}\right)\left(\eta_{1}+2 k+\eta_{2}\right)+2\left(\eta_{1}+k\right)\left(\eta_{2}+k\right) \log \left[\frac{k+\eta_{1}}{k+\eta_{2}}\right]\right]}{\left(\eta_{1} \eta_{2}\right)^{2}\left(2 k+\eta_{1}+\eta_{2}\right)\left[36+M_{1}\left\{6(3+C) K 1^{2}+3(3+C) k\left(\eta_{1}+\eta_{2}\right)+4 C\left(\eta_{1}^{2}+\eta_{1} \eta_{2}+\eta_{2}^{2}\right)\right\}\right]} \\
& +\frac{36(1+C) M_{1}\left(\eta_{1} \eta_{2}\right)\left[\left(Q+\eta_{2} \eta_{1}\right)\left(\eta_{1}+\eta_{2}+2 k\right)+2\left(\eta_{1}+k\right)\left(\eta_{2}+k\right) \log \left[\frac{k+\eta_{1}}{k+\eta_{2}}\right]\right]}{12 M_{1}\left(\eta_{1}+k\right)^{2}\left[2 C\left(\eta_{2} \eta_{1}\right)\left(\eta_{2}+k\right)^{2}+\left(\eta_{2}+k\right)^{2} 3(1+C) k \log \left[\frac{k+\eta_{1}}{k+\eta_{2}}\right]\right] \log \left[\frac{k+\eta_{1}}{k+\eta_{2}}\right]} .
\end{aligned}
$$



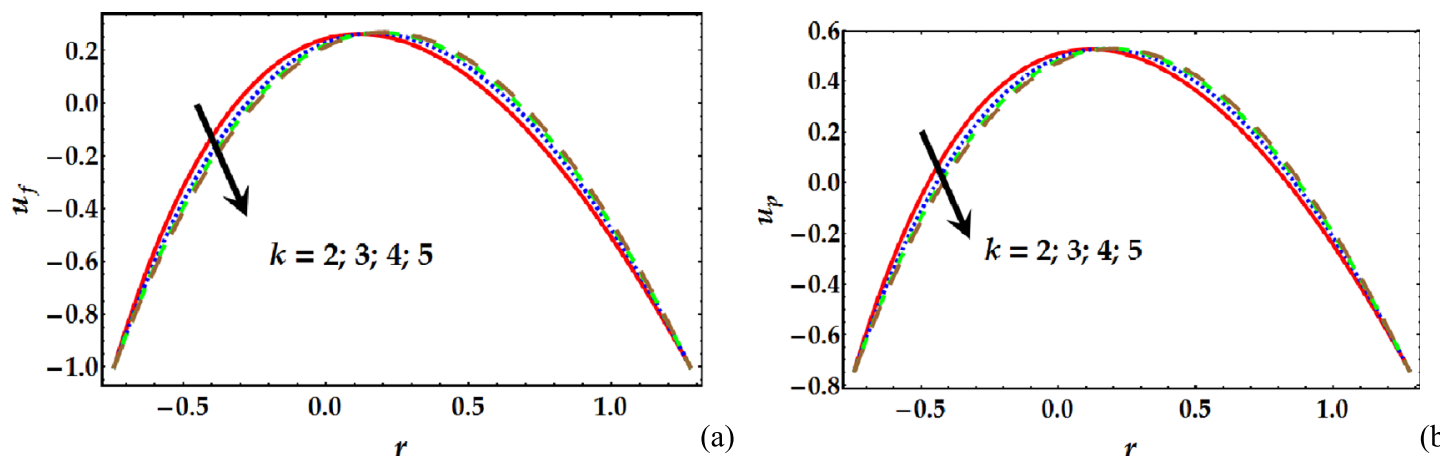

Fig 3. Velocity curves for multiple values of $k$.
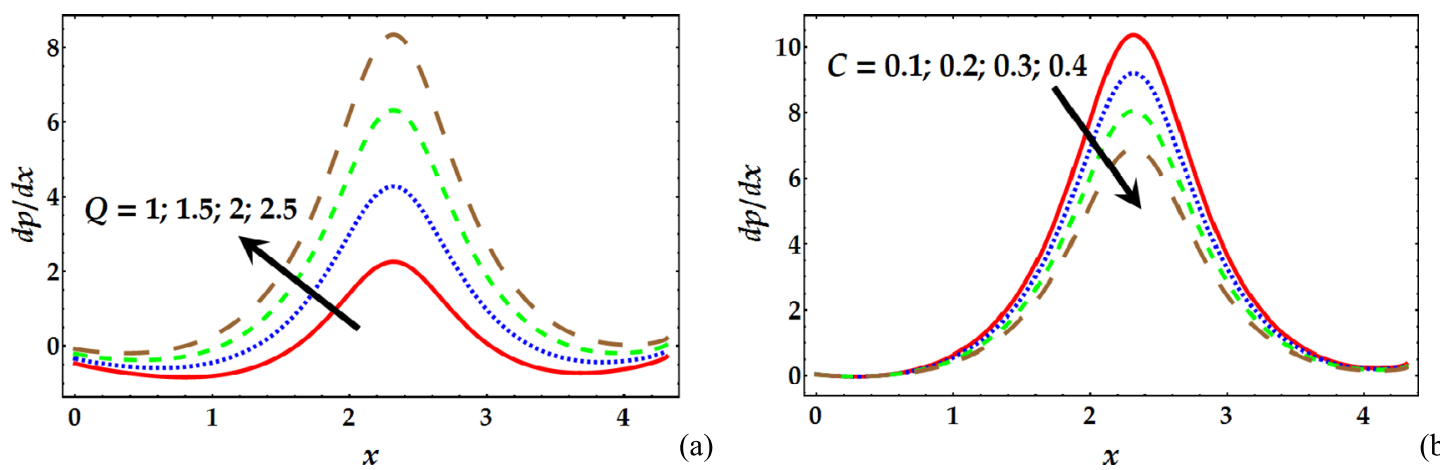

Fig 4. Pressure gradient for multiple values of $Q$ and $C$.

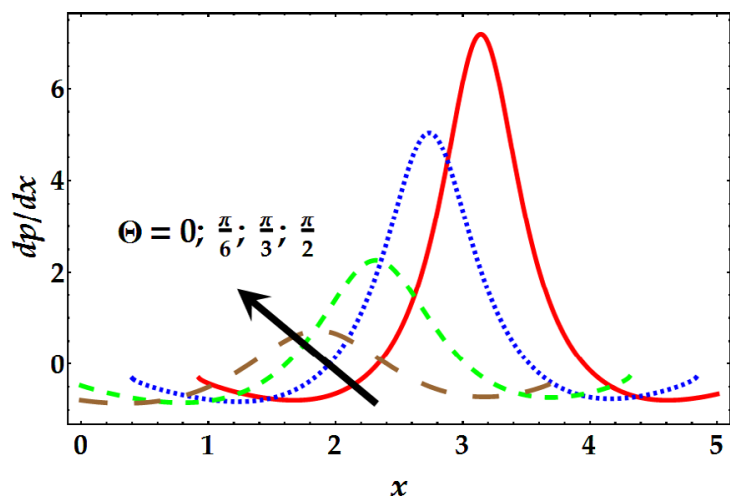

Fig 5. Velocity curves for multiple values of $\Theta$.

\subsection{Temperature distributions}

Figure $7 \mathrm{a}$ and $\mathrm{b}$ and Figure 8 represent the temperature distributions and consist of the present results obtained in equation (27). From Figure 7a and b, it is concluded that particle volume fraction " $C$ " and curvature parameter " $k$ " markedly enhance the temperature profile. Figure 8 shows the variation of Prandtl number " $P_{r}$ " and Eckert number " $E_{c}$ " on temperature distribution. In this figure, we can see that both the parameters (i.e., Eckert number " $E_{c}$ " and Prandtl number " $P_{r}$ ") enhance the temperature. The ration between momentum diffusivity to thermal diffusivity is known as Prandtl number. Inspection of Figure 8 also reveals that when Prandtl number is high, then thermal diffusivity is more prominent on momentum diffusivity. It is worth mentioning here that such type of results is substantial for those fluids which have a large Prandtl number and less significant for low Prandtl number fluids such as ionized gasses etc.

\subsection{Concentration distributions}

Figures 9-11 are plotted for concentration distributions to see the physical effects of involved parameters. It can be viewed from Figure 9a that concentration distribution significantly diminishes due to a more significant influence of particle volume fraction. However, for large values of curvature parameter, concentration distribution markedly rises (see Fig. 9b). From Figure 10 we can see that higher values of Eckert number and Prandtl number produce a marked reduction in a concentration distribution. Figure 11 is plotted for " $N_{A}=\left(S_{c} S_{r}\right)$ " (product of Schmidt number " $S_{c}$ " and a Soret number " $S_{r}$ ". Schmidt number " $S_{c}\left(=\frac{\mu_{s}}{\rho D_{m}}\right)$ " is the ratio between momentum and mass diffusivity and it is beneficial to determine the convection process of mass and momentum diffusion. This figure reveals that an increment in " $N_{A}$ " tends to resist the concentration distribution. It happens because mass diffusivity becomes more dominated over momentum diffusivity and due to higher Soret number, the particles move to cold region from hot region and as a result, the concentration distribution diminishes.

\subsection{Trapping mechanism}

This subsection deals with the trapping phenomena which are observed through the streamlines. Streamlines represent the "family of curves" that are promptly tangent 

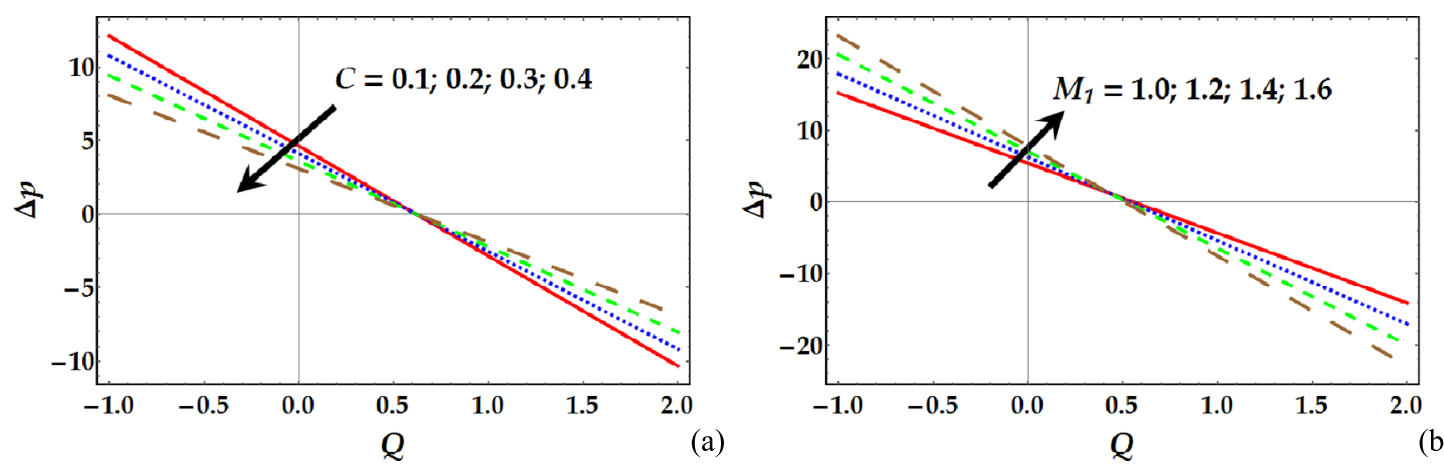

Fig. 6. Pressure rise vs. volume flow rate for various values of $C$ and $M_{1}$.
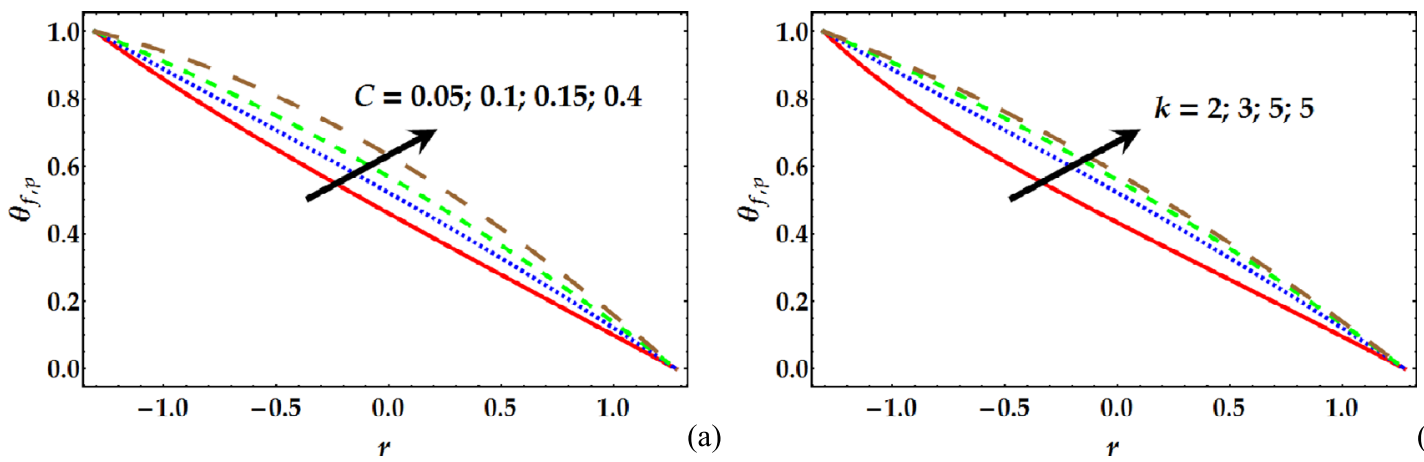

Fig 7. Temperature profile for multiple values of $C$ and $k$.

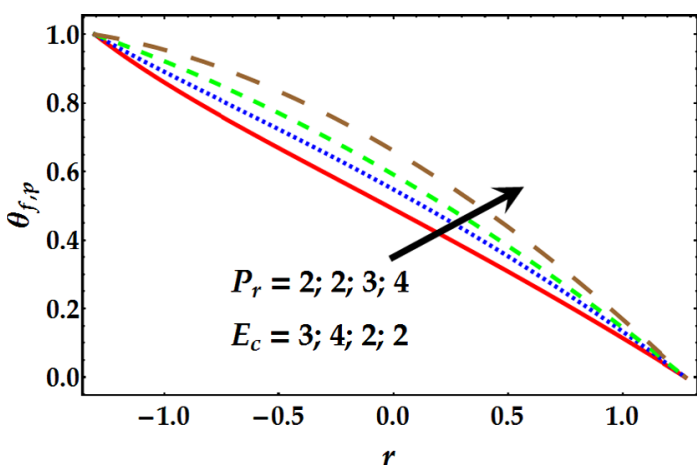

Fig 8. Temperature profile for multiple values of $P_{r}$ and $E_{c}$.

to velocity vectors of a flow. Moreover, it is composed of bolus circulating inside. The composition of bolus circulating the fluid is embedded by numerous streamlines that are known as "Trapping". These inner circulating boluses propagate along the peristaltic wave. This mechanism is beneficial in blood flow situations, i.e., composition of thrombus and propagation of food bolus in a gastrointestinal tract. Figures 12-14 depict the streamlines for multiple values of associated parameters in momentum equations. It can be seen from Figures 12 and 13 that by increasing particle volume fraction " $C$ " and volumetric flow rate " $Q$ " the number of streamlines reduces significantly. However, we have observed that the number of streamlines increases very slowly with the increment in curvature parameter " $k$ ".

\section{Concluding remarks}

The viscous particle-fluid ("two-phase") model has been used to analyse the simultaneous impact of mass and heat transfer on peristaltically induced motion through an asymmetric curve channel. The exact solution expressions of velocity, concentration, pressure gradient and temperature are obtained whereas numerical integration has been carried to explore the pumping features. Graphical illustrations are presented against multiple values of involved several parameters. The critical findings are described below

- there is a critical value of $r$ around which the velocity distribution acts opposite;

- pressure gradient enhances due to the increment in volume flow rate and phase difference;

- pressure gradient tends to diminish significantly for large values of $C$;

- peristaltic pumping and retrograde pumping region decreases for higher values of $C$ and opposite for suspension parameter $M_{1}$;

- Prandtl number, particle volume fraction, and Eckert number have an increasing impact on temperature profile;

- curvature parameter fails to provide a significant resistance temperature distribution, while its attitude is converse for concentration profile;

- concentration profile acts in similar form against $C N_{A}$ (product of $S_{r} S_{c}$ ). 

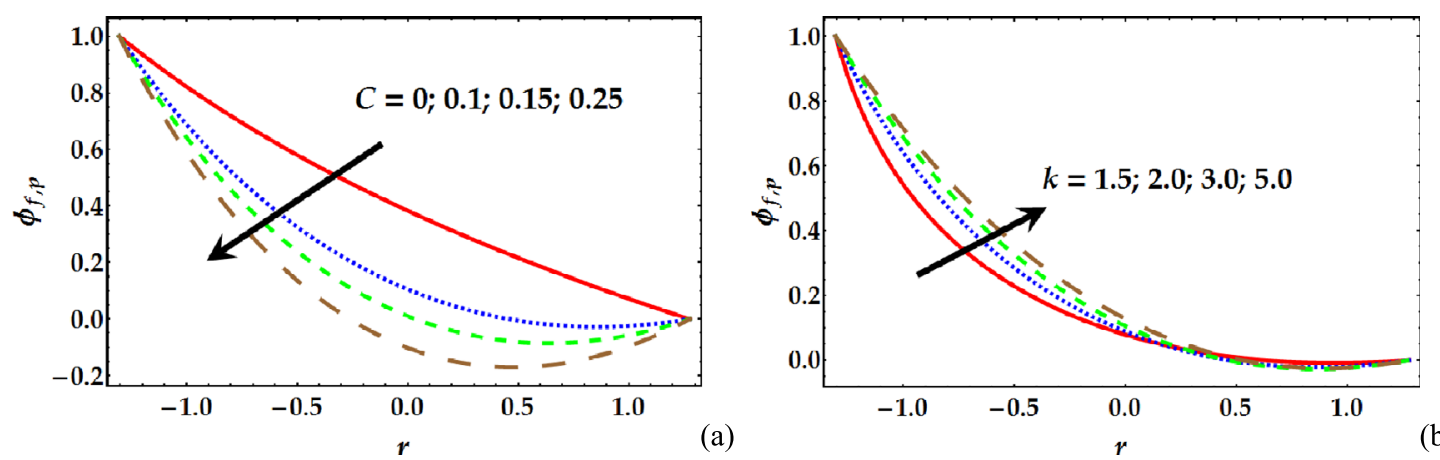

Fig 9. Temperature profile for multiple values of $C$ and $k$.

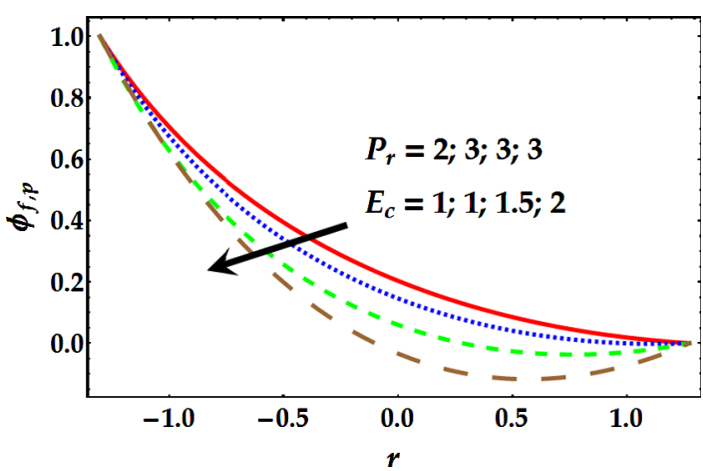

Fig 10. Concentration profile for multiple values of $P_{r}$ and $E_{c}$.

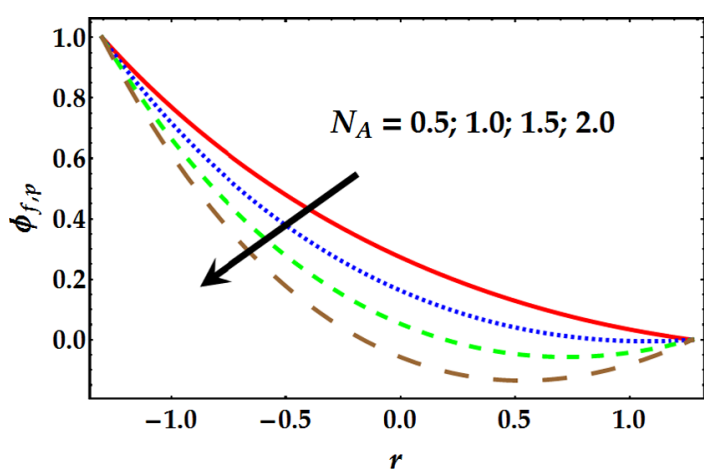

Fig 11. Concentration profile for multiple values of $N_{A}$.
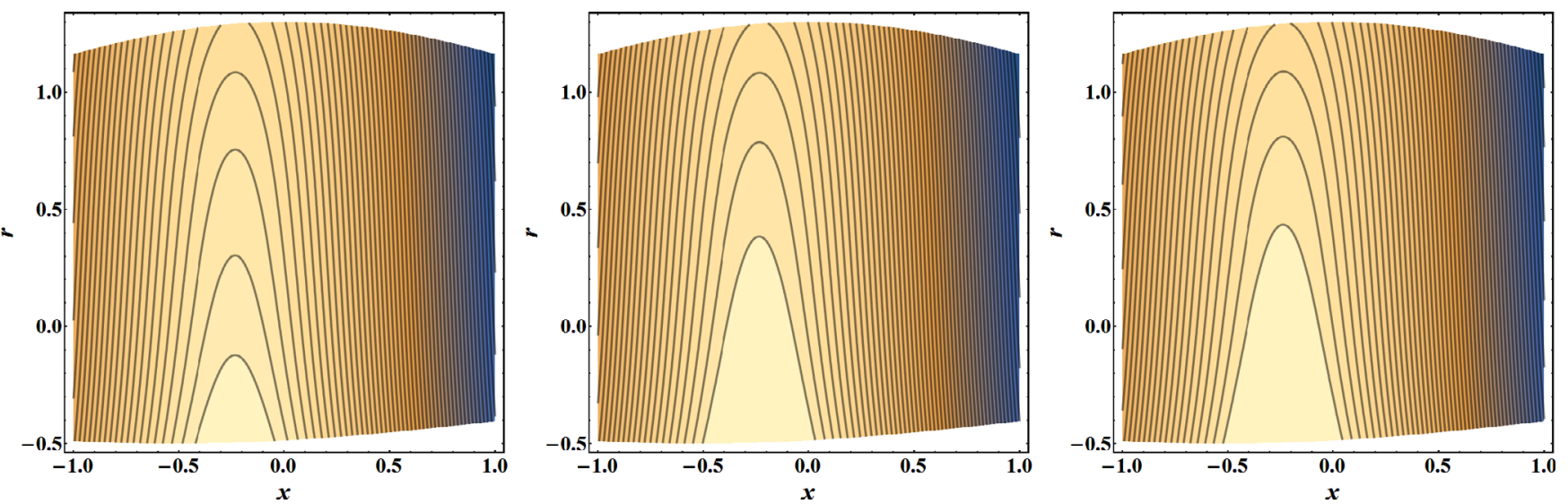

Fig. 12. Graph of stream lines for multiple values of $C=0.1,0.2,0.3$.

The present study has neglected rheological working fluids. It will be examined in the next studies with different biological non-Newtonian fluids. Moreover, the present study addresses various engrossing results and characteristics that warrant further investigation on two-phase flow model. The subject reveals a platform for the mathematical formulation, and hopefully it will help further to examine the two-phase non-Newtonian fluids experimentally with mass and heat transfer.

\section{Nomenclature}

$u, v \quad$ Velocity components

$T_{m} \quad$ Mean temperature
$M_{1} \quad$ Suspension parameter

$D_{m} \quad$ Mass diffusivity

$k_{T} \quad$ Thermal diffusivity

$E_{c} \quad$ Eckert number

$P \quad$ Pressure

$R^{*} \quad$ Radius of curvature

$P_{r} \quad$ Prandtl number

$C \quad$ Volume fraction density

$\tilde{t} \quad$ Time

Re Reynolds number

$S_{c} \quad$ Schmidt number

$C_{p} \quad$ Effective heat capacity

$S_{r} \quad$ Soret number

$K \quad$ Thermal conductivity

$F \quad$ Concentration 

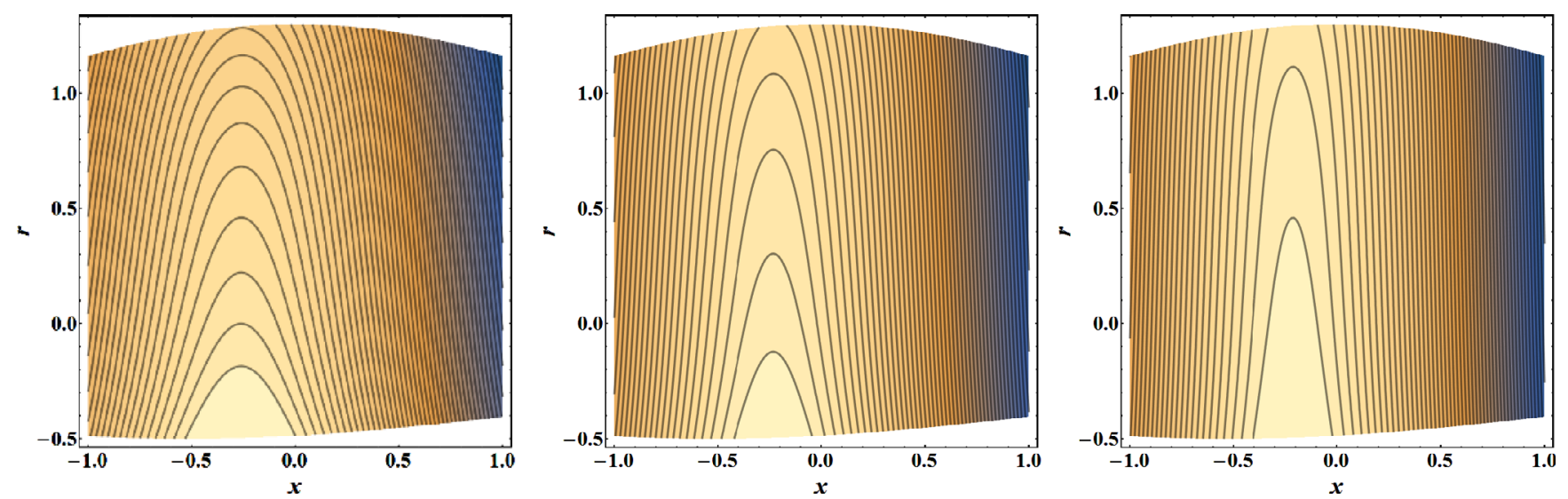

Fig. 13. Graph of stream lines for multiple values of $Q=0.2,0.4,0.6$.
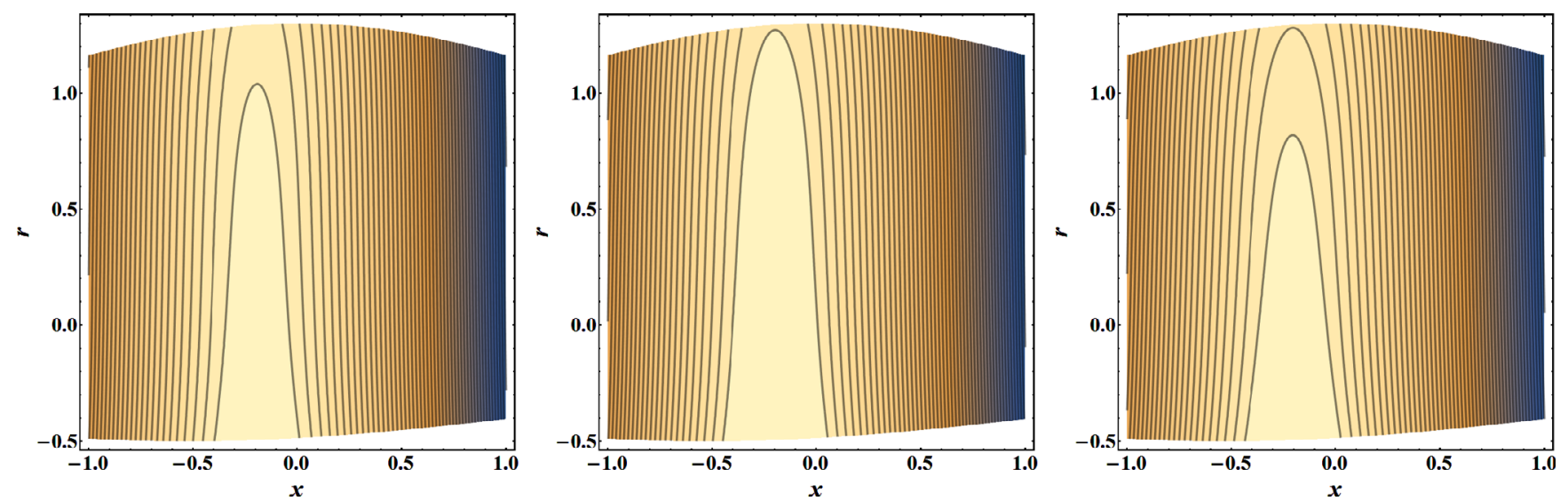

Fig. 14. Graph of stream lines for multiple values of $k=0.5,0.6,0.7$.

$T \quad$ Temperature

$S \quad$ Drag coefficient

$Q \quad$ Volume flow rate

$d_{1}+d_{2}$ Channel width

$a_{1}, b_{1} \quad$ Waves amplitudes

$k \quad$ Curvature parameter

c Wave celerity

\section{Greek symbols}

\section{$\theta_{f, p} \quad$ Temperature}

$\varpi_{T}$ Thermal equilibrium time

$\mu_{s} \quad$ Viscosity of the fluid

$\Theta \quad$ Phase difference

$\phi_{f, p}$ Concentration

$\lambda$ Wavelength

$\rho \quad$ Fluid density

$\varpi_{v} \quad$ Relaxation time of the particle

$\phi \quad$ Stream function

\section{Subscripts}

$p$ Particulate phase

$f$ Fluid phase

\section{References}

[1] M. Mishra, A.R. Rao, Peristaltic transport of a Newtonian fluid in an asymmetric channel, Z. Angew. Math. Phys. 54 (2003) 532-550

[2] K.S. Mekheimer, Non-linear peristaltic transport through a porous medium in an inclined planar channel, J. Porous Media 6 (2003)

[3] M.V. Reddy, M. Mishra, S. Sreenadh, A.R. Rao, Influence of lateral walls on peristaltic flow in a rectangular duct, J. Fluids Eng. 127 (2005) 824-827

[4] M.A.A. Elnaby, M.H. Haroun, A new model for study the effect of wall properties on peristaltic transport of a viscous fluid, Commun. Non-linear Sci. Numer. Simul. 13 (2008) $752-762$

[5] T. Hayat, M. Javed, A.A. Hendi, Peristaltic transport of viscous fluid in a curved channel with compliant walls, Int. J. Heat Mass Transf. 54 (2011) 1615-1621

[6] J.V. Ramanamurthy, K.M. Prasad, V.K. Narla, Unsteady peristaltic transport in curved channels, Phys. Fluids 25 (2013) 091903

[7] S. Nadeem, A. Riaz, R. Ellahi, Peristaltic flow of viscous fluid in a rectangular duct with compliant walls, Comput. Math. Model. 25 (2014) 404-415

[8] J.C. Misra, S.K. Pandey, Peristaltic transport of a particlefluid suspension in a cylindrical tube, Comput. Math. Appl. 28 (1994) 131-145 
[9] M. Saxena, V.P. Srivastava, Particulate suspension flow induced by sinusoidal peristaltic waves, Jpn. J. Appl. Phys. 36 (1997) 385

[10] K.S. Mekheimer, E.F. El Shehawey, A.M. Elaw, Peristaltic motion of a particle-fluid suspension in a planar channel, Int. J. Theor. Phys. 37 (1998) 2895-2920

[11] K.S. Mekheimer, Y. Abd Elmaboud, A.I. Abdellateef, Particulate suspension flow induced by sinusoidal peristaltic waves through eccentric cylinders: thread annular, Int. J. Biomath. 6 (2013) 1350026

[12] M.H. Kamel, I.M. Eldesoky, B.M. Maher, R.M. Abumandour, Slip effects on peristaltic transport of a particle-fluid suspension in a planar channel, Appl. Bionics Biomech. 2015 (2015)

[13] S. Akram, K.S. Mekheimer, Y.A. Elmaboud, Particulate suspension slip flow induced by peristaltic waves in a rectangular duct: effect of lateral walls, Alex. Eng. J. 57 (2018) 407-414

[14] M.M. Bhatti, A. Zeeshan, M.M. Rashidi, Influence of magnetohydrodynamics on metachronal wave of particlefluid suspension due to cilia motion, Eng. Sci. Technol. Int. J. 20 (2017) 265-271

[15] M.M. Bhatti, A. Zeeshan, N. Ijaz, Slip effects and endoscopy analysis on blood flow of particle-fluid suspension induced by peristaltic wave, J. Mol. Liq. 218 (2016) 240-245

[16] S.I. Abdelsalam, K. Vafai, Particulate suspension effect on peristaltically induced unsteady pulsatile flow in a narrow artery: blood flow model, Math. biosci. 283 (2017) 91-105

[17] D. Tripathi, A mathematical model for swallowing of food bolus through the oesophagus under the influence of heat transfer, Int. J. Therm. Sci. 51 (2012) 91-101

[18] S. Srinivas, R. Muthuraj, J. Sakina, A note on the influence of heat and mass transfer on a peristaltic flow of a viscous fluid in a vertical asymmetric channel with wall slip, Chem. Ind. Chem. Eng. Q. 18 (2014) 483-493

[19] R. Ellahi, M.M. Bhatti, K. Vafai, Effects of heat and mass transfer on peristaltic flow in a non-uniform rectangular duct, Int. J. Heat Mass Transf. 71 (2014) 706-719

[20] M.M. Bhatti, A. Zeeshan, R. Ellahi, Heat transfer analysis on peristaltically induced motion of particle-fluid suspension with variable viscosity: clot blood model, Comput. Methods Prog. Biomed. 137 (2016) 115-124

[21] M.M. Bhatti, A. Zeeshan, Heat and mass transfer analysis on peristaltic flow of particle-fluid suspension with slip effects, J. Mech. Med. Biol. 17 (2017) 1750028

[22] M.M. Bhatti, A. Zeeshan, N. Ijaz, O.A. Bég, A. Kadir, Mathematical modelling of nonlinear thermal radiation effects on EMHD peristaltic pumping of viscoelastic dusty fluid through a porous medium duct, Eng. Sci. Technol. Int. J. 20 (2017) 1129-1139

[23] D.A. Drew, Mathematical modeling of two-phase flow, Annu. Rev. Fluid Mech. 15 (1983) 261-291

Cite this article as: A. Zeeshan, N. Ijaz, M.M. Bhatti, Flow analysis of particulate suspension on an asymmetric peristaltic motion in a curved configuration with heat and mass transfer, Mechanics \& Industry 19, 401 (2018) 


\section{Appendix}

$$
\begin{aligned}
& u_{0}=\frac{2 k\left(\eta_{1}-\eta_{2}\right)\left[6+C\left(-6+d p / d x\left\{3 k\left(\eta_{1}+\eta_{2}\right)+2\left(\eta_{1}^{2}+\eta_{1} \eta_{2}+\eta_{2}^{2}\right)\right\}\right)\right]}{6(-1+C)\left(\eta_{1}-\eta_{2}\right)\left(2 k+\eta_{1}+\eta_{2}\right)} \\
& +\frac{d p}{d x} \frac{3(-1+C) k\left[\left(k+\eta_{1}\right)^{2} \eta_{2} \log \left[\eta_{1}+k\right]\left(\eta_{2}+2 k\right)-\left(2 k \eta_{1}+\eta_{1}^{2}\right)\left(\eta_{2}+k\right)^{2} \log \left[\eta_{2}+k\right]\right]}{6(-1+c)(\eta 1-\eta 2)(2 K 1+\eta 1+\eta 2)}, \\
& u_{1}=\frac{\left(-\eta_{2}+\eta_{1}\right)\left[3+C\left\{-3+d p / d x\left(-3 k^{2}+\eta_{1}^{2}+\eta_{1} \eta_{2}+\eta_{2}^{2}\right)\right\}\right]}{3(-1+c)\left(\eta_{1}-\eta_{2}\right)\left(2 k+\eta_{1}+\eta_{2}\right)} \\
& +\frac{3(1-C) k^{2} d p / d x\left[\left(k+\eta_{1}\right)^{2} \log \left[\eta_{1}+k\right]-\left(\eta_{2}+k\right)^{2} \log \left[\eta_{2}+k\right]\right]}{3(-1+c)\left(-\eta_{2}+\eta_{1}\right)\left(2 k+\eta_{1}+\eta_{2}\right)}, \\
& u_{2}=\frac{d p}{d x} \frac{-2 C\left(\eta_{1}-\eta_{2}\right)\left(2 k+\eta_{1}+\eta_{2}\right)+3(1-C)\left[k\left(k+\eta_{1}\right)^{2} \log \left[\eta_{1}+k\right]-\left(\eta_{2}+k\right)^{2} k \log \left[\eta_{2}+k\right]\right]}{6(-1+C)\left(\eta_{1}-\eta_{2}\right)\left(2 k+\eta_{1}+\eta_{2}\right)}, \\
& u_{3}=\frac{C}{3(1-C)}, u_{4}=\frac{d p}{d x} \frac{k^{3}\left((-1+C)\left(\eta_{1}-\eta_{2}\right)\left(\eta_{1}+\eta_{2}\right)+2 k\left((-1+C) \eta_{1}+\eta_{2}-C \eta_{2}\right)\right)}{2(-1+C)\left(\eta_{1}-\eta_{2}\right)\left(2 k+\eta_{1}+\eta_{2}\right)}, \\
& u_{5}=k^{2} \frac{d p}{d x}, u_{6}=\frac{k}{2} \frac{d p}{d x}, \\
& \theta_{0}=\frac{\left(4+C E_{c} M_{1}(d p / d x)^{2} P_{r} \eta_{2}\left(2 k+\eta_{2}\right)\right) \log \left[k+\eta_{1}\right]-C E_{c} M_{1}(d p / d x)^{2}\left(2 P_{r} \eta_{1} k+P_{r} \eta_{1} \eta_{1}\right) \log \left[k+\eta_{2}\right]}{4\left(\log \left[\eta_{1}+k\right]-\log \left[\eta_{2}+k\right]\right)}, \\
& \theta_{1}=\frac{-4+C E_{c} M_{1}(d p / d x)^{2} P_{r}\left(\eta_{1}-\eta_{2}\right)\left(2 k+\eta_{1}+\eta_{2}\right)}{4\left(\log \left[k+\eta_{1}\right]-\log \left[k+\eta_{2}\right]\right)}, \theta_{2}=-\frac{1}{2} C E_{c} M_{1} k\left(\frac{d p}{d x}\right)^{2} P_{r}, \theta_{3}=-\frac{1}{2} C E_{c} M_{1}\left(\frac{d p}{d x}\right)^{2} P_{r}, \\
& \phi_{0}=\frac{-\left(-4+C E_{c} M_{1} N_{A}(d p / d x)^{2} P_{r} \eta_{2}\left(2 k+\eta_{2}\right)\right) \log \left[k+\eta_{1}\right]-C E_{c} M_{1}(d p / d x)^{2} N_{A} P_{r} \eta_{1}\left(2 k+\eta_{1}\right) \log \left[k+\eta_{2}\right]}{4\left(\log \left[k+\eta_{1}\right]-\log \left[k+\eta_{2}\right]\right)}, \\
& \phi_{1}=\frac{-4+C E_{c} M_{1} N_{A}(d p / d x)^{2} P_{r}\left(\eta_{1}-\eta_{2}\right)\left(2 k+\eta_{1}+\eta_{2}\right)}{4\left(\log \left[k+\eta_{1}\right]-\log \left[k+\eta_{2}\right]\right)}, \phi_{2}=-\frac{N_{A}}{2} C E_{c} M_{1} k\left(\frac{d p}{d x}\right)^{2} P_{r}, \phi_{3}=-\frac{N_{A}}{2} C E_{c} M_{1}\left(\frac{d p}{d x}\right)^{2} P_{r},
\end{aligned}
$$

